\title{
Proceedings of the 14th Liquid and Amorphous Metals International Conference
}

\author{
Editors: T. Scopigno, ${ }^{1}$ S. De Panfilis ${ }^{1,2}$, and A. Di Cicco, ${ }^{3,4}$ \\ ${ }^{1}$ Dipartimento di Fisica, Università di Roma "La Sapienza”, I-00185 Roma (Italy). \\ ${ }^{2}$ Centro Studi e Ricerche e Museo Storico della Fisica E. Fermi, I-00184 Roma (Italy). \\ ${ }^{3}$ CNISM, Sezione di Fisica, Scuola di Scienze e Tecnologie, Università di Camerino, I-62032 Camerino \\ (MC), Italy (permanent address). \\ ${ }^{4}$ Institut de Minéralogie et de Physique des Milieux Condensés, UMR 7590, CNRS, Universités Paris \\ 6 et Paris 7, Paris, France.
}

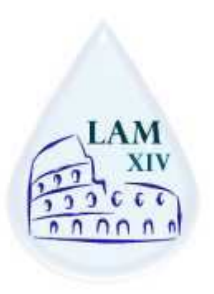

\author{
XIV Liquid and Amorphous Metals Conference \\ Rome $11-16$ july 2010 \\ "Sapienza" - University of Rome
}

\begin{abstract}
PREFACE
The 14th Liquid and Amorphous Metals International Conference (LAM14) was held on 11-16 July 2010 in Rome, Italy. About 200 scientists from several countries all around the world attended this conference, which is part of a series started in 1966 at Brookhaven and continued in the last decades on a regular three-year basis (the last two Conferences were held in Metz, 2004 and Ekaterinburg, 2007). In line with the traditions of this conference, the participation of junior researchers and graduate students was encouraged and young researchers played an active role in the conference. This conference series is mainly devoted to discuss the state-of-the-art in the field of liquid and amorphous metals and alloys. However, several communications on new trends for advanced studies of materials including some non-metallic systems have been included as a part of the scientific program. In particular, the organizers solicited communications about updated research results on the breakthroughs and advances from the cutting-edge facilities at many synchrotron radiation, $4^{\text {th }}$ generation, and neutron sources.

There were no parallel sessions and all oral reports were arranged into three groups: invited talks (45 $\mathrm{min}$ ), keynotes (35 $\mathrm{min}$ ) and oral contributions $(25 \mathrm{~min}$ ). The program included 7 sessions on different topics with 10 invited speakers and 4 keynote talks. The sessions were split into several subsessions covering the five mornings and four afternoons for a total of 64 oral communications. The structure of the conference was slightly changed with respect to the previous editions of the conference, aiming to give a strong emphasis to hot and advanced topics emerging in the field.

The session about "Dynamics" began with the invited talk of W. Schirmacher who reviewed recent results about atom dynamics in liquid metals in light of the mode coupling theory. This session included 9 talks about new experimental and theoretical results on the dynamics not only of molten metals, but also of oxides and ionic melts.
\end{abstract}




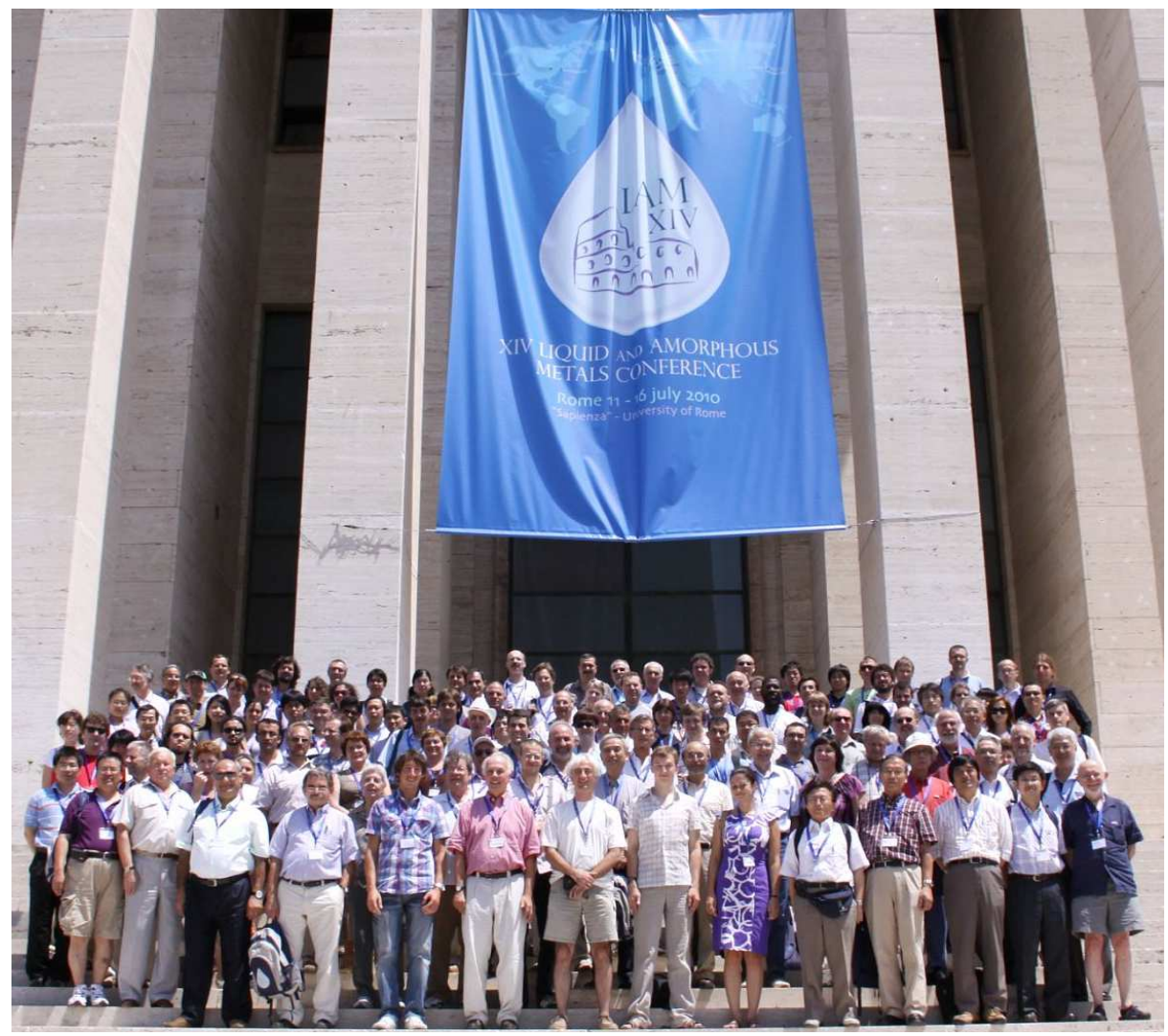

Fig. 1. Participants of the LAM14 conference gathered in front of the main entrance of the Rectorate of the University "La Sapienza" in Roma.

The session about "Extreme conditions" ran over two different days of the conference, in view of the number and quality of contributions received. Three invited talks introduced the different subsessions, covering important activities in this growing field. A. Filipponi reported about recent works carried out using synchrotron radiation on undercooled liquids also under high-pressure conditions, I. Egry reviewed the current state-of-the-art for levitation methods applied to measurements of atomic structure and dynamics, and finally J. I. Raty discussed recent results about ab-initio molecular dynamics calculations on molten alkali metals. The "Extreme conditions" session included a total of 18 oral communications covering different aspects of the current research in this field.

The "Structure and Thermodynamics" session was also placed on two different days of the conference and included two invited talks of F. Shimojo, reporting about large-scale simulations of nanostructured materials using the Density Functional theory, and K. Matsuda, talking about synchrotron radiation studies of expanded alkali metals. The session included a total of 18 talks dealing with various experimental and theoretical studies of structure and dynamics in liquids and glasses.

An afternoon session about "Surface and Interfaces" was devoted to the main contributions in this field. The session was opened by the invited talk of P. Pershan, reviewing the current state-of-the-art on X-ray scattering studies of liquid metal surfaces. The session included a total of 6 talks reporting current experimental and simulation studies in this field.

An entire morning session was devoted to forefront research in "Ultrafast Phenomena", a field now growing fast associated with new experimental possibilities coming from sub-picosecond lasers and forthcoming 4th generation free-electron-laser sources. The dynamics of photo-induced melting has been discussed in the invited talk given by R. Ernstorfer. A second invited talk by S. L. Johnson was devoted to recent time-resolved pump-probe X-ray diffraction studies of ultrafast heated materials. A 
total of 7 talks reported about the current state-of-the-art in the application of ultrafast techniques to liquid and amorphous metals.

Finally, a session about "Glasses and Clusters" began with an invited talk by A. L. Greer reporting about detailed studies of the localization of shear in metallic glasses. The session included a total of 8 talks on recent experimental and theoretical studies of the structure and other peculiar properties of nanostructured materials and glasses.

Moreover, the poster sessions were particularly lively and interesting and included more than 100 posters displayed for the whole duration of the Conference. A best poster prize of 400 Euro was awarded to Dr. M. Çolakogullari from the Physics Department of the Trakya University (Edirne, Turkey) for his work entitled: "An investigation of the local structure and dynamic properties of undercooled liquid silicon using the orbital-free ab-initio molecular dynamics method".

It was decided during the joint meeting of both the International Advisory Board and International Program Committee that the next conference in the LAM series, LAM15, will be arranged by Prof. Haozhe Liu from Natural Science Research Center, Academy of Fundamental and Interdisciplinary Sciences in HIT (now in the International Advisory Committee of LAM). Prof. Chuang Dong from Dalian University of Science and Technology will co-chair the next LAM conference in China in 2013.

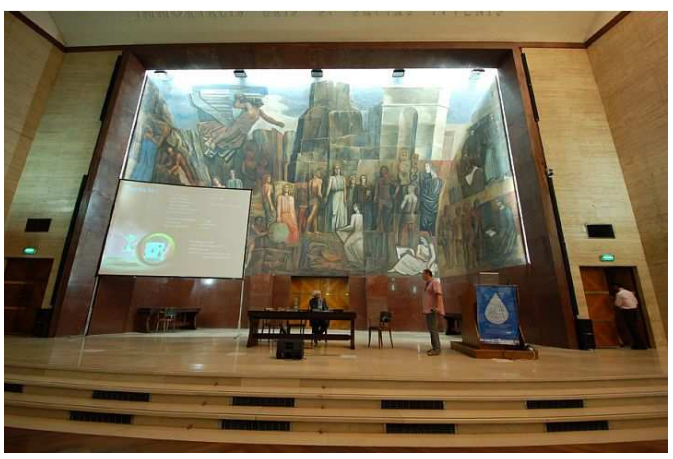

Fig. 2. Plenary talk in the aula magna of "La Sapienza".

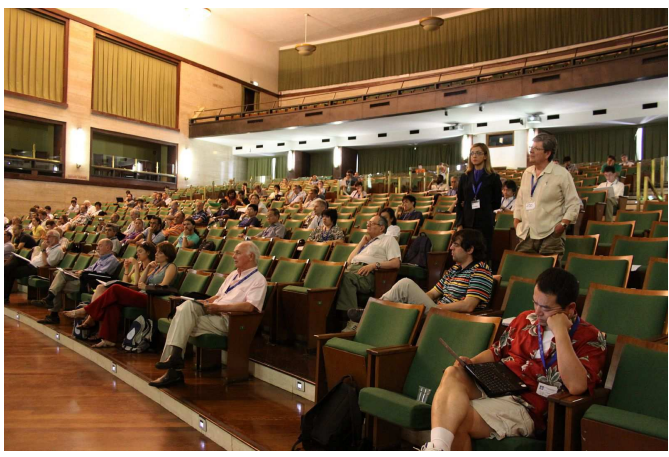

Fig. 3. Question time during the oral sessions.

We are confident that the participants have appreciated the good University infrastructures and experienced personnel, and enjoyed the visit of the ancient town of Roma as well as the original social program proposed during the Conference.

About 260 abstracts were received and 75 manuscripts were finally submitted for publication on the European Journal of Physics: Web of Conference. The articles which are collected in this volume were accepted after standard peer-reviewing. A group of about 20 outstanding scientists in the field contributed to the review process and in many cases suggested revisions of the manuscripts to improve scientific presentations matching the needs for a publication on an international journal. We thus hope this work will constitute a useful reference for the ongoing scientific activity in the field.

LAM14 was also a nice opportunity to discuss the state-of-the-art and emerging frontiers in the fields covered by this LAM Conference. Consequently, we invited a number of scientists leading the field to contribute to a dedicated issue of the European Journal of Physics: Special Topics.

We would like to express our gratitude and appreciation to the sponsors of this Conference for their generous support: Dipartimento di Fisica of the University of Roma "La Sapienza", the ELETTRA synchrotron radiation facility (Trieste, Italy), ENEA (Italy), Sparx (INFN-LNF, Frascati, Italy), Coherent Inc. (USA), Crisel Instruments srl (Italy).

Finally, we would like to acknowledge the committees, the organizing staff and in particular its core group whose efforts made the LAM14 conference a success.

Roma, Italy, March 8th, 2011

The proceedings editors,

Tullio Scopigno, Simone De Panf lis and Andrea Di Cicco 
EPJ Web of Conferences

\section{COMMITTEES AND STAFF}

\section{Chairs}

Tullio Scopigno and Andrea Di Cicco

\section{International advisory committee}

$\begin{array}{ccc}\text { J. Bermejo (Spain) } & \text { M. Calvo-Dahlborg (France) } & \text { A. Di Cicco (Italy) } \\ \text { C. Dong (China) } & \text { J.G. Gasser (France) } & \text { B. Gelchinski (Russia) } \\ \text { D. Gonzalez (Spain) } & \text { P. Hausler (Germany) } & \text { K. Hoshino (Japan) } \\ \text { W.-C. Pilgrim (Germany) } & \text { P. Popel (Russia) } & \text { D.L. Price (France) } \\ \text { P. Salmon (UK) } & \text { T. Scopigno (Italy) } & \text { K. Tamura (Japan) } \\ \text { K. Tsuji (Japan) } & \text { M. Yao (Japan) } & \end{array}$

\section{International Program committee}
A.C. Barnes (UK)
K.F. Kelton (USA)
M. Mendelev (USA)
D. Holland-Moritz (Germany)
A. Mikula (Austria)
K. Khishchenko (Russia)
P.A. Madden (UK)
J. Morris (USA)
M.L. Saboungi (France)
V. Sidorov (Russia)
A. P. Tsai (Japan)

\section{Local organizing committee}
G. Angilella
G. Aquilanti
L. Battezzati
M. Celino
S. De Panfilis
A. Filipponi
C. Masciovecchio
C. Petrillo
E. Principi
G. Ruocco
F. Sacchetti
A. Trapananti

\section{Heads of the Conference Secretariat}

F. Lupinacci P. Pasolini

\section{Technical Support}

S. Cazzato F. Medici M. Perciballi 